\title{
Apparent friction and cohesion of a partially wet granular material in steady-state shear
}

\author{
H. Louati *, D. Oulahna, A. de Ryck \\ Centre RAPSODEE, Mines Albi, CNRS, Université de Toulouse, Campus Jarlard, F-81013 Albi Cedex 09, France \\ haithem.louati@mines-albi.fr driss.oulahna@mines-albi.fr (D. Oulahna), alain.deryck@mines-albi.fr (A. de Ryck).
}

\begin{abstract}
A B S T R A C T
The shear resistance of glass beads with wetting liquid incorporated (polyethylene glycol of molecular weight of $400 \mathrm{~g} / \mathrm{mol}$ ) is experimentally and theoretically investigated in a large range of both liquid content (from $0.007 \%$ to $20 \%$ in volume) and normal loading, in a steady state flowing situation. It is observed that the behaviour is not purely frictional (shear stress proportional to the normal stress) except for the lowest liquid content (up to $0.1 \%$ ) for which it is observed that the friction coefficient of wet granular material is slightly higher than the dry mate rial. At high normal loading, an apparent cohesion is observed and the behaviour may be interpreted as frictional, with the same friction coefficient plus an additional normal stress due to the capillary forces between the beads. At low normal loading, the apparent cohesion is less and may be qualitatively explained by the diminution of bro ken capillary contacts during shearing when the porosity of the granular bed increases. This result is corroborated by a theoretical approach estimating the capillary forces in play.
\end{abstract}

Shear test

Wet granular material

Granular friction

Cohesion

Capillary force

\section{Introduction}

Many powder processing methods such as granulation or coating re quire humid environments. The presence of liquid affects the properties of the granular material. One common problem is to evaluate the flow resistance of wet powder during transport, mixing and emptying pro cesses. This can be caused by very small amounts of liquid (for example condensed airborne humidity).

Hornbaker et al. (1997) [1] reported that the changes in behaviour of granular material induced by moisture are primarily caused by adhesive forces associated with interstitial liquid bridges between grains. For dry granular material, adhesive forces are the results of inter particle forces, mainly Van der Waals and electrostatic forces. In the latter case the attractive forces are long range forces and cannot be considered as inde pendent adhesive forces. Van der Waals' forces are created between permanent dipoles, induced dipoles or permanent and induced dipoles. These forces depend on the size of the particles and the distance between them. Electrostatic forces are created by differential electrical potentials of particles surfaces caused by friction between particles and the wall of the equipment being used [2].

For wet granular materials, capillary forces prevail due to the liquid bridges formed between particles. Depending on the amount of liquid in wet granular material, different states of liquid saturation have been distinguished. Newitt and Conway (1958) [3] introduced the notions of pendular, funicular and capillary states. In the pendular state, with small quantities of liquid, particles are held together through separated liquid bridges formed at the contact points. Particles are attracted to each other due to the surface tension of the liquid and the capillary pressure. An increase in the saturation of the liquid leads to a transitional situation, the so called funicular state, in which the liquid partially fills the spaces between particles. The capillary state is reached when all available spaces are filled with liquid. Another state of adsorp tion layers preceding the pendular one was introduced by Pietsch [4], where liquid is bonded to the particle surface. These adsorption layers can increase inter particle forces such as Van der Waals forces.

We focus here on the adsorption layers and on the pendular states. For monodisperse particles, the pendular state prevailed when the volumic liquid saturation $\mathrm{S}$ was lower than $25 \%$. This corresponds to a liquid volume of $17 \%$ of the volume of the solid, for the monodisperse porosity $\varepsilon=0.41$ ( $S$ is defined as the volume of pores filled by liquid rel ative to the total pore volume [5]).

Many authors have studied the mechanical influence of the presence of liquid in granular material in the pendular state. Rumpf (1962) [6] studied the relationship between tensile strength and liquid bridge forces, and proposed a model to estimate this tensile strength. This model will be used in this study to evaluate the capillary forces acting on the wet monodispersed glass beads. Hornbaker et al. (1997) [1], Fraysse et al. (1999) [7] and Bocquet et al. (2002) [8] focused on the stat ic properties of powder. They demonstrated the influence of humidity on the angle of repose. They observed that a small quantity of liquid in creases the value of this angle. The latter is related to the maximal ratio between shear and normal stresses. In these studies the flow of granular material occurs at low levels of normal stress since they are located close to the free surface. This is not the case in the quasi static and 
dynamic states studies performed in shear cells or mixers, where the applied or established normal stresses may influence the flow proper ties $[9,10]$. However, these studies focus on the yield criterion rather than steady state situation in the case of quasi static experiments in shear cells or are not able to quantify or vary the normal stresses in steady state flows in mixers. None of these articles explores the rela tionship between steady state shear and normal stresses for different normal loadings when liquid is added.

In this paper, we focus on the effects of both liquid content and load ing on the shearing behaviour of wet granular materials, using glass beads of $70110 \mu \mathrm{m}$ in diameter, in steady state low velocity shear sit uations. Experiments are performed for a wide range of normal stress (from 0.3 to $11 \mathrm{kPa}$ ) and liquid content (from 0.007 to $20 \%$ in volume).

\section{Materials and methods}

\subsection{The wet granular bed and its porosity}

The granular media used consists of glass beads provided by SOVITEC, with diameter sieve ranging from $70 \mu \mathrm{m}$ to $110 \mu \mathrm{m}$. They have a spherical shape (sphericity index $\psi=0.96$ ) and a smooth sur face. The true density of glass beads is $\rho_{\mathrm{s}}=2.47 \mathrm{~g} / \mathrm{cm}^{3}$ and the poros ity in dry conditions is $\varepsilon=0.41$.

Glass beads were wetted using polyethylene glycol of $400 \mathrm{~g}$ molar weight $\left(\mathrm{PEG}_{400}\right)$ from Alfa Aesar. This liquid was chosen since it wets the glass beads well and has a low volatility. The other characteristics are the density $\rho_{\mathrm{L}}=1.128 \mathrm{~g} / \mathrm{cm}^{3}$ at $20^{\circ} \mathrm{C}$; viscosity $\eta=99.01 \mathrm{mPa} \mathrm{s}$ at $25^{\circ} \mathrm{C}$ and the surface tension $\gamma=56.74 \mathrm{mN} / \mathrm{m}$ at $23.5^{\circ} \mathrm{C}$. $\mathrm{PEG}_{400}$ is also slightly hygroscopic, non toxic and nonhazardous.

Small amounts of PEG $_{400}$ were added to glass beads. Thirteen samples of wet glass beads were prepared by doubling the quantity of liquid each time starting from $27.5 \mu \mathrm{l} / \mathrm{kg}$. Then, the wet glass beads were mixed to ensure the distribution of the liquid in the gran ular material (using a Kenwood mixer). The concentration of liquid is expressed by a volume ratio $V_{R}$ which represents the volume of liq uid relative to the volume of particles.

In order to study the influence of liquid saturation on the density configuration of wet glass beads, the mean bulk density $\rho$ (weight of particles divided by the total apparent volume) and the wet porosity (the volume of the void relative to the total volume of the wet granular material)

$\varepsilon_{\text {wet }} \quad 1-\frac{\rho}{\rho_{S}} \frac{1+V_{R}}{1+V_{R}\left(\frac{\rho_{L}}{\rho_{S}}\right)}$,

were estimated before starting the shear test, and consolidated with a normal stress of $12 \mathrm{kPa}$ in another apparatus.

Fig. 1 shows the variation of the porosity of the bed as a function of the volume ratio $V_{R}$ of liquid added on a semi logarithmic scale. For a small addition of liquid, the porosity of the glass beads decreases slight ly. This decrease is possibly due to the lubrication at some contact points between particles, allowing denser configurations.

When $V_{R}$ increases beyond $0.05 \%$, a clear increase in porosity is observed. This transition is due to the capillary forces. Indeed, when the concentration of liquid reaches a certain value, the capil lary forces exceed the weight of the particles. As a consequence, the liquid bridges created between particles are able to sustain a loose assembly of particles, with larger gaps in the granular material leading to a lower density configuration. Then, the porosity tends to wards saturation when $V_{R}$ reaches between $1 \%$ and $7 \%$, where the in crease in liquid content may contribute to an increase in the volume of liquid bridges without greatly affecting the porosity. The decrease of the wet porosity beyond $7 \%$ of $V_{R}$ is explained by the filling of the inter particle spaces by the liquid.

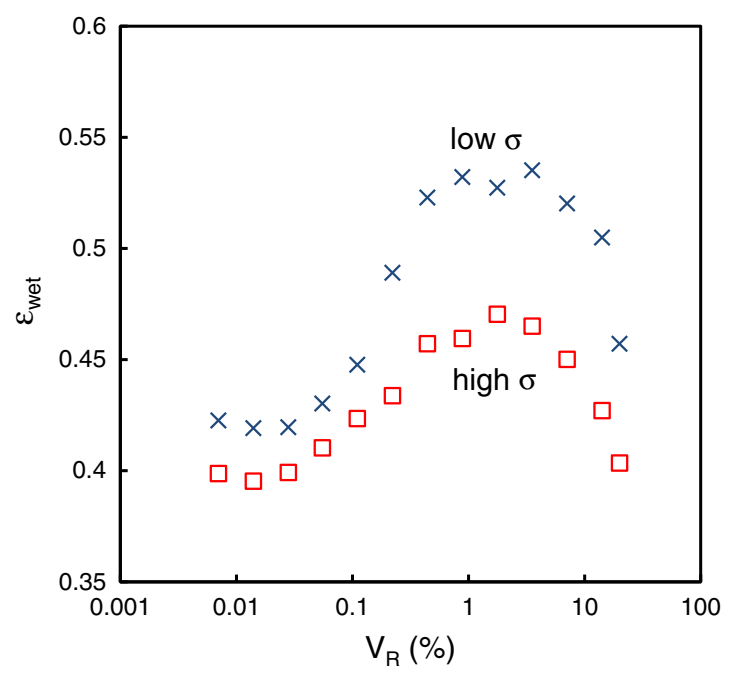

Fig. 1. Mean porosity versus the liquid volume ratio for the different samples at the filling of the shear cell (low $\sigma$ ) or pre-consolidated at $12 \mathrm{kPa}$ (high $\sigma$ ).

The relationship between porosity, capillary forces and liquid content was studied by Feng and Yu (1998) and Yu et al. (2003) $[11,12]$. They found that the dry porosity of glass beads of different sizes $(250,1000,2000$ and $4000 \mu \mathrm{m})$ increases with the liquid con tent to reach a maximum value at a critical point. This relationship may be explained by the creation of liquid bridges when adding liq uid to the granular material which induces a capillary force. This limits the particle motion to form a packing and therefore rises the porosity. Above the critical point, the saturation of the capillary forces also leads to a saturation of porosity. They also assumed that an increase in the amount of liquid added (beyond the maximum quantities) results in capillary forces vanishing and, thus, to a de crease in porosity.

The porosity of wet glass beads has also been studied for the same maximal normal stress (about $12 \mathrm{kPa}$ ) used in the shear experiments, in order to examine the loading effect on the porosity. Fig. 1 compares them with the porosities at low normal stress. It shows the same varia tion of the $\varepsilon V_{R}$ curves (slight decrease, leap, saturation and decrease), but with a lower magnitude. This result shows that this application of normal stress did not prevent the increase of the porosity caused by the capillary forces.

\subsection{The shear test in steady state conditions}

A Schulze shear cell was used to study the flow properties of the wet glass beads (see Fig. 2). This equipment consists of an annular trough with an inner diameter of $10 \mathrm{~cm}$ and an outer diameter of $20 \mathrm{~cm}$. Nor mal force $\mathrm{N}$ is applied through an annular lid, so as to exert a normal stress. A counterweight system exerts an against force $F_{A}$ that is used to balance the weight of the lid and other parts connected to it in order to be able to obtain low normal stresses [13].

To run a shear test, the shear cell is filled with the sample and rotates at a constant rotational velocity $\omega$ of $2.3 \mathrm{mrad} / \mathrm{s}$. The bottom of the trough and the lower side of the lid are rough due to the presence of the teeth which prevent wall slippage. The shear plane occurs below the teeth of the lid, therefore the weight of the particles between the teeth is taken into consideration when calculating the normal stress $\sigma$ acting on the sheared surface. We used:

$$
\sigma \quad \frac{M g}{A}+\rho g h_{t}
$$

where $M$ is the normal loading weight, $g$ is the gravitational constant, $A$ is the area of the lid and $h_{t}=4 \mathrm{~mm}$ is the height of the teeth. The value 


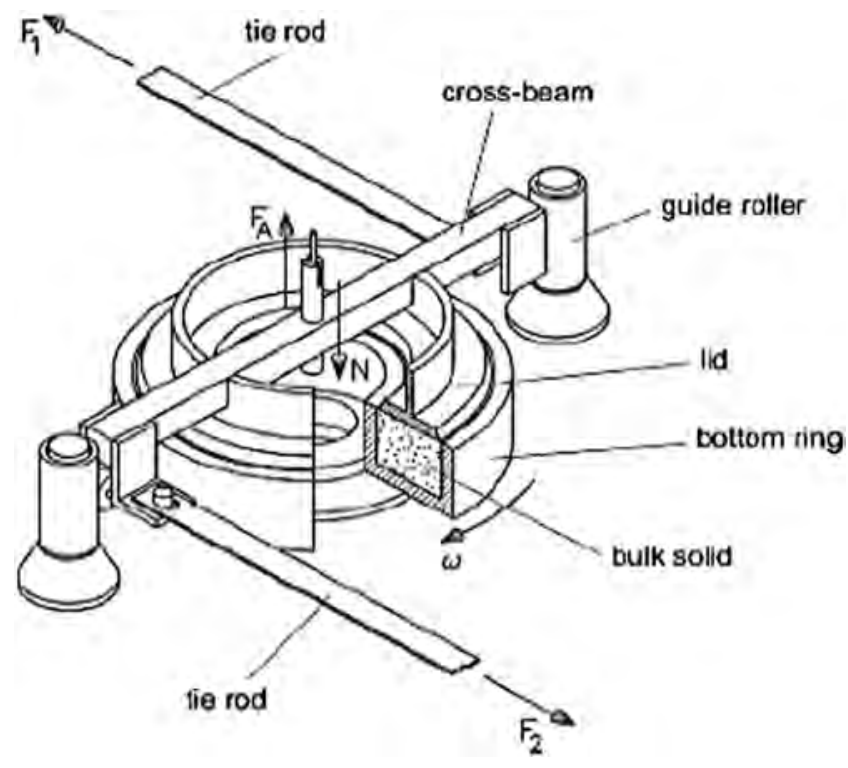

Fig. 2. Schematic representation of the Schulze cell [13].

chosen for the apparent density $\rho$ is that observed after the filling of the annular cell, disregarding any volume variation due to the normal load ing since the second term of Eq. (2) is small compared to the stress due to the loading mass in our experiments.

The shearing process consists of measuring the mean tangential stress $\tau$ from the torque applied by the rotating powder on the powder kept fixed together with the lid; and obtained by the two force sensors $\left(F_{1}\right.$ and $F_{2}$ ), which hold it (see Fig. 2 ). This measurement is repeated for various normal stresses.

These experiments were performed for the dry and wet glass beads with various concentration of $\mathrm{PEG}_{400}$. For each sample, a range of normal loads were used from $24 \mathrm{~kg}$ to $0.5 \mathrm{~kg}$ by removing $0.5 \mathrm{~kg}$ at each step. For a given normal load, the sample was sheared for approximatively $1 \mathrm{~min}$. After a peak (not studied here), a plateau value or an oscillatory steady state regime was recorded as shown in Fig. 3.

In the following section, the steady state shear stress is deduced from the diagram (shear stress time) and then used to study the flow properties of wet glass beads.

\subsection{The steady state shear stress signal}

For dry glass beads, the response of the shear stress signal is con stant and reaches a plateau value for all the normal stresses applied, as shown in Fig. 3 left. This is not the case for wet glass beads sheared at a sufficiently high normal stress $(\sigma>2 \mathrm{kPa})$, for which an oscilla tory stick slip motion takes place. Such a stick slip motion may be observed with a velocity weakening friction coefficient if the granu lar bed shear apparatus is not stiff enough $[14,15]$.

The oscillatory behaviour of the shear stress at a steady state is treated by computing the mean value $\tau_{s}$ and the standard deviation $\Delta \tau$. Fig. 4 shows the mean signal (circles) and $\tau_{s} \pm \Delta \tau$ for the sample with a volume ratio of $0.055 \%$, as it is one of the largest amplitudes of oscillation. As can be seen in the figure, $\Delta \tau$ increases in an approxi mately linear relationship with normal stress to reach $0.4 \mathrm{kPa}$ at about $\sigma=10 \mathrm{kPa}$, after a threshold value of normal stress of around $2 \mathrm{kPa}$. The shear stress oscillates within a maximum range of $10 \%$, and consequently only the mean value will be used afterwards. In the same Fig. 4, it may be observed that this mean value should be close to the constant value that would be observed for a stiffer sys tem, since it remains close to that extrapolated from the behaviour at low normal stress (continuous line).

\section{Results and discussion}

\subsection{Effect of moisture on the steady state flow}

We will now investigate the effect of a small amount of liquid on the steady state flow. The glass beads, ranging from 70 to $110 \mu \mathrm{m}$ in diam eter, are non cohesive in dry conditions. The shear resistance is induced by the friction between the particles. In wet conditions, the shear resis tance is also caused by the inter particle forces, which also modify the texturization of the granular bed, as indicated by the porosity measure ments (Fig. 1).

Fig. 5 illustrates the steady state mean shear stress $\tau_{s}$ versus the nor mal stress $\sigma$ for various liquid contents. This figure shows only part of the range of applied normal stresses (from 0 to $6 \mathrm{kPa}$ ), for better visual ization of the curves. We can observe that $\tau_{s}$ is dependent on the liquid content as the curves are shifted relative to the dry case: the shear stress of wetted glass beads increases when the concentration of liquid increases.

In this present study, the shear resistance is also studied for a wide range of applied stresses, allowing us to check whether the
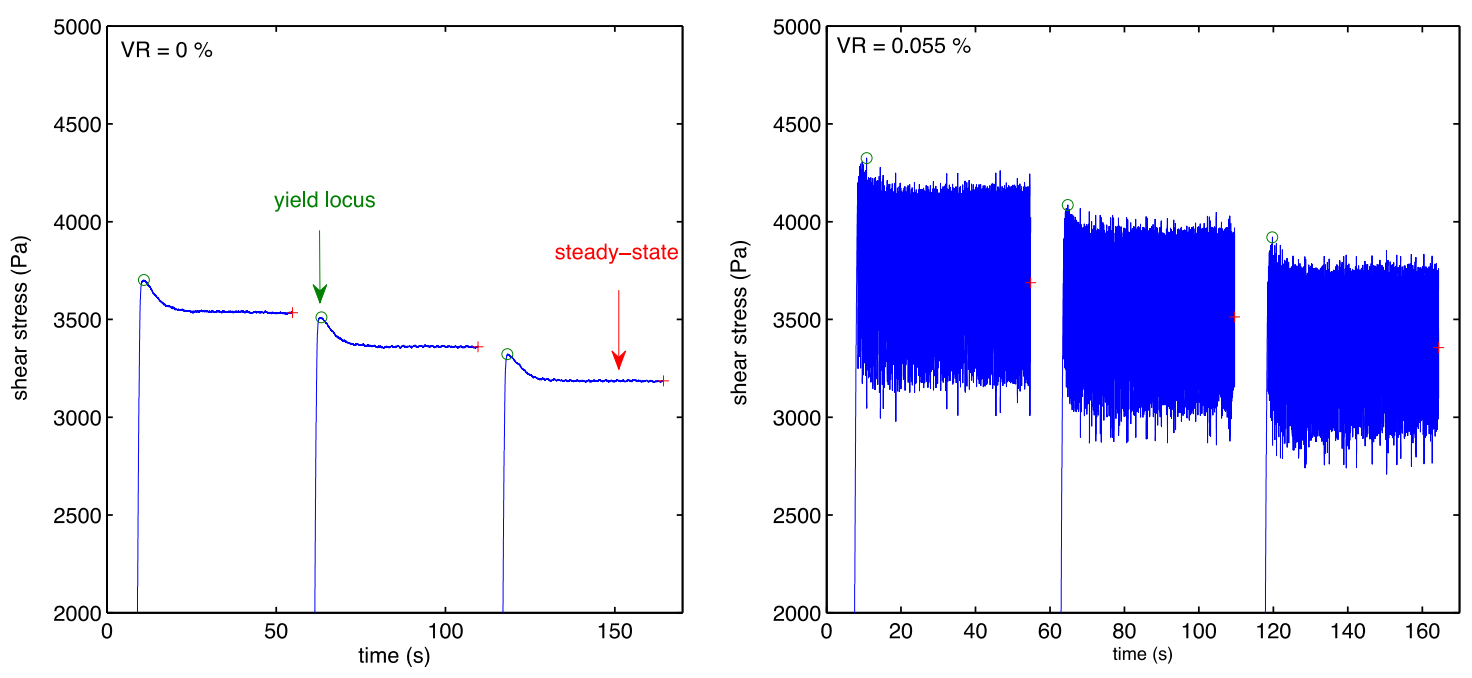

Fig. 3. Shear stress versus time for 3 normal stresses. Left: dry case. Right: wet case with $V_{R}=0.55 \%$ ). 


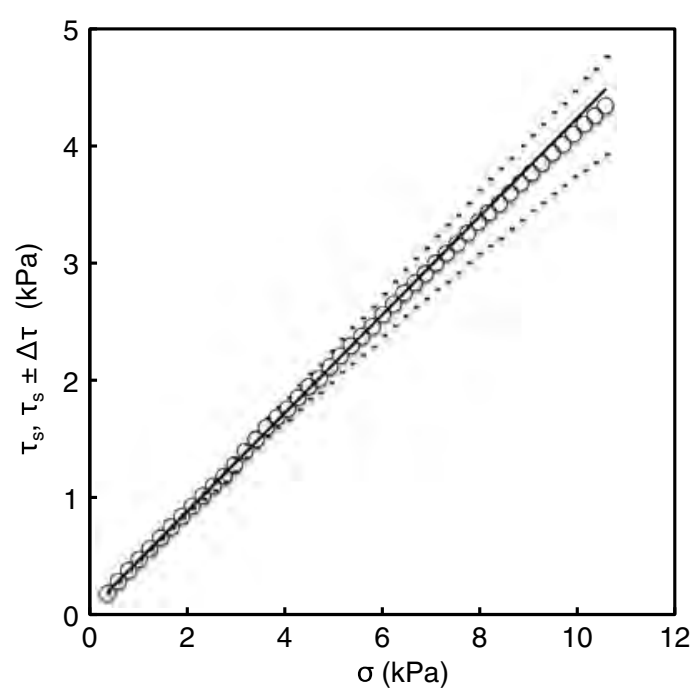

Fig. 4. Mean shear stress $\tau_{s}$ (circles) and $\tau_{s} \pm \Delta \tau$ (bars) plotted against $\sigma$ for a liquid volume ratio of $0.55 \%$. The straight line is the extrapolated linear behaviour without stickslip instability.

apparent friction $\tau_{s} / \sigma$ remains constant for a given liquid content or whether it also depends on the normal stress applied. For this pur pose, enhancing the differences between the curves and focusing on the relative changes when introducing a given quantity of liquid, we define the relative shear stress $\tau_{r}$ by subtracting the shear stress in dry conditions from the $\tau_{s}$ measured for wet conditions:

$\tau_{r} \tau_{s}-\mu_{d r y} \sigma$.

$\tau_{r}$ is plotted versus $\sigma$ in Fig. 6 for the full range of normal stresses in vestigated (up to $11 \mathrm{kPa}$ ).

The observed curves show a clear variation of the shape depending on the normal stress and the liquid content, and may be classified with in the following regimes:

a) For small liquid content If the volume ratio of liquid is small, rang ing from $0.07 \%$ o to $0.55 \%$, the observed behaviour is frictional. The steady state shear stress is proportional to the normal stress: $\tau_{s}=$ $\mu_{\text {wet }} \sigma$, where $\mu_{\text {wet }}$ is the friction coefficient of the wet granular mate rial. The observed curves show that the friction of slightly wet glass

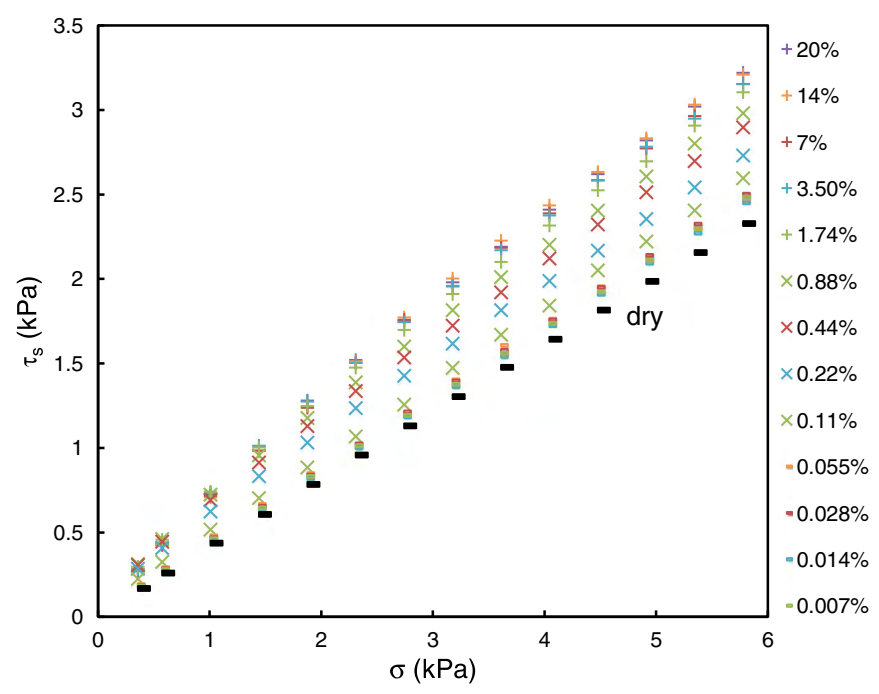

Fig. 5. $\tau_{s}$ as a function of $\sigma$ (limited to $6 \mathrm{kPa}$ ) for dry glass beads and wet glass beads with a volume ratio $V_{R}$ ranging from $0.07 \%$ o to $20 \%$.

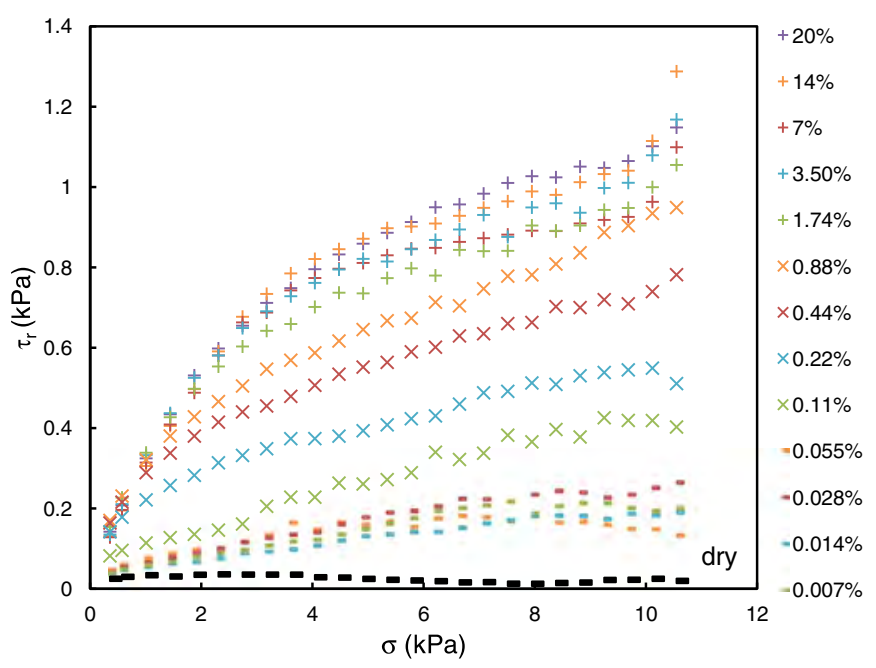

Fig. 6. Relative shear stress $\tau_{r}$ as a function of $\sigma$ for the same range of liquid volume ratio as shown in Fig. 5.

beads is a little higher than that observed for the dry case; the fric tion coefficient determined from curves $\tau_{s} \sigma$ in Fig. 5 or 6 is equal to 0.42 instead of the 0.40 of dry glass beads, with no significant de pendency on $V_{R}$ within the range from $0.07 \%$ o to $0.55 \%$ since the curves are superimposed.

When the concentration of liquid increases further (beyond $0.11 \%$ by volume), the curves show a slope failure, and data can be fitted using two straight lines, depending on the applied normal stress. Hereinaf ter, the experimental results obtained will be explored separately at high and low normal stresses.

b) Higher liquid content, high normal stress For $\sigma>4 \mathrm{kPa}$, the shear normal stress relationship is linear, with a slope close to $\mu_{\text {wet, }}$, that which is observed in the $a$ ) regime, but shifted upward in the $\tau_{r} \sigma$ plane when the liquid content is increased, defining residual cohe sion $c$ or apparent traction $T_{S}$ as:

$$
\tau_{s} \approx \mu_{w e t} \sigma+c\left(V_{R}\right) \approx \mu_{w e t}\left(\sigma+T_{s}\left(V_{R}\right)\right)
$$

This enhanced shear resistance originates from the inter particle forces due to the presence of liquid and may be interpreted as if these forces exert a supplementary pressure $T_{S}$ on the shear plane. Fig. 7 shows traction $T_{s}$ versus $V_{R}$ on a semi logarithmic scale. In the following section, the values obtained will be compared to a the oretical prediction based on the estimation of the capillary forces be tween the particles.

c) Higher liquid content, low normal stress For lower normal stresses, the shear resistance is observed to be below that extrapolated at high normal stress $(b)$ and slightly greater than that for the lowest liquid content $(a)$. In this regime, the shear resistance saturates or presents a maximum value for a given normal loading, when the liq uid content is increased.

The apparent cohesion or traction remains low compared to the ex trapolated one (Fig. 7) while the apparent friction coefficient $\mu_{\text {wet }}$ de duced from the slope of the curves $\tau_{s} \sigma$ is much greater than that observed in the b) regime. The local slope of the shear normal stresses curve increases from approximately 0.42 to 0.6 and then tends towards saturation.

This phenomenon is explained qualitatively by the fact that the po rosity at low normal stress is higher than at high normal stress. There fore, there is less contact between particles, fewer particles to separate in the shear plane, and there is more rolling accomodation of the shear deformation which avoids breaking half of the contacts per 


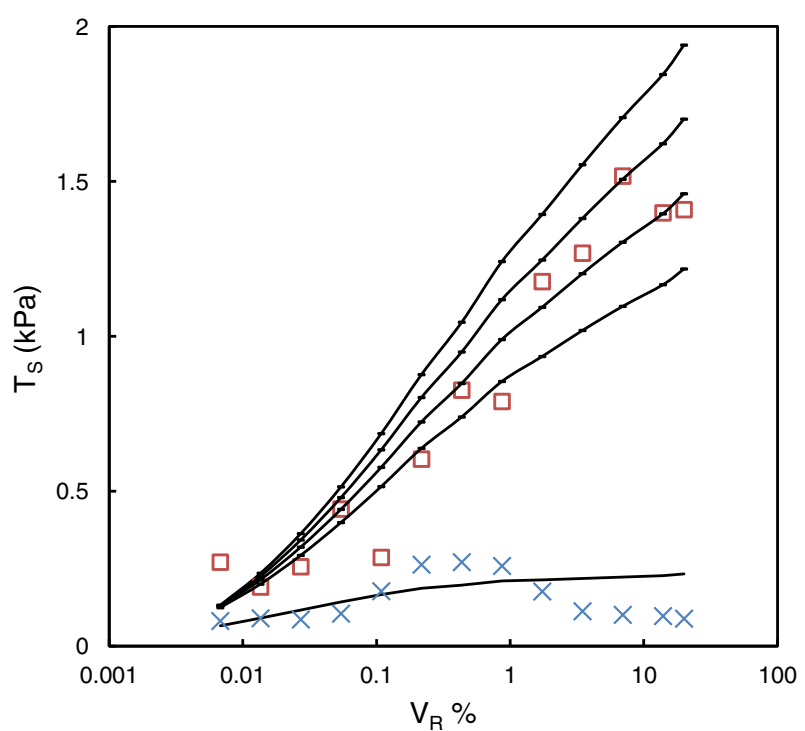

Fig. 7. Experimental and theoretical traction (tensile strength) as a function of the volume ratio on a semi-logarithmic scale. Squares and crosses: experimental data for high normal stress $(\sigma>4 \mathrm{kPa})$ and low normal stress $(\sigma<4 \mathrm{kPa})$ respectively. Continuous lines: theoretical traction for various coordination numbers $(1,5,6,7$ and 8$)$ shown from bottom to top in this figure.

particle on the shear plane (see Fig. 9). This point will be described more quantitatively in the next section.

\section{Theoretical estimation of the tensile strength using the Rumpf model}

The tensile strength $T_{s}$ represents the tensile force at failure divided by the cross section of the agglomerate. In the case of a random packing of monosized spherical particles in the pendular state, the tensile strength may be approximated by the Rumpf relation [5] as follows:

$$
T_{S} \quad \frac{1-\varepsilon_{\text {wet }}}{\pi} \kappa \frac{F_{\text {cap }}}{d^{2}} .
$$

For wet granular media, the tensile strength is caused by the sum of liquid bridge forces $F_{\text {cap }}$ at contact points between the particles apart from the failure plane. The above equation also depends on the particle size $d$, the wet porosity $\varepsilon_{\text {wet }}$ and the average coordination number $\kappa$. The latter may be empirically related to $\varepsilon_{\text {wet }}$ according to this relationship [4]:

$\kappa \pi / \varepsilon_{\text {wet }}$,

leading to a coordination number between 7 and 8 for dense packing and around 6 to 7 for loose packing.

Many models have been developed to determine the capillary force due to the presence of a liquid meniscus between two spheres. Fischer (1926) [16] proposed a model based on the toroidal approximation (the liquid bridge is assimilated to an arc of circle). It allows the capillary force for spherical particles with smooth surface to be estimated. This force is expressed as the sum of the surface tension integrated on the contour plus the capillary depression integrated on the meniscus area [17]. Another approach, developed by Adams and Perchard (1985), the so called boundary method, consists in evaluating these forces at the sphere surface [ 18 20].

When the separation distance between the particles is zero, these approaches predict a decrease in the capillary force with an increasing volume of liquid, which does not correspond to our experimental obser vations. Pietsch (1968) [21] proposed to resolve the decrease of the cap illary force, adding a minimum value corresponding to the separation distance $2 \mathrm{a}$ between two spheres, caused by the asperities on the sur face of the particles and allowing the liquid to be incorporated in be tween (see Fig. 8a).

An approximate value of the minimum half separation distance $a$ was evaluated from scanning electron microscopy (SEM) by mea suring the height of the asperities on the glass bead surface. Fig. 8b shows an example of a SEM image showing two glass beads touch ing, in the presence of a small amount of liquid $\left(V_{R}=0.11 \%\right)$. The observation of the particle surface shows several asperities of a sig nificant size. The half separation distance was estimated to be be tween 1 and $2 \mu \mathrm{m}$. This value is similar to other observations of the roughness of a $100 \mu \mathrm{m}$ glass bead by atomic force microscopy (AFM), where the height of the surface roughness is approximately $1 \mu \mathrm{m}[22]$. A dimensionless half separation distance, $\delta=a / R$, has been set to 0.03 and considered to be constant for all the experiments.

The capillary force was subsequently determined using the Adams and Perchard approach which states [19]

$F_{\text {cap }} \quad 2 \pi R \gamma \sin ^{2} \alpha+\pi R^{2} \sin ^{2} \alpha \Delta P$, a

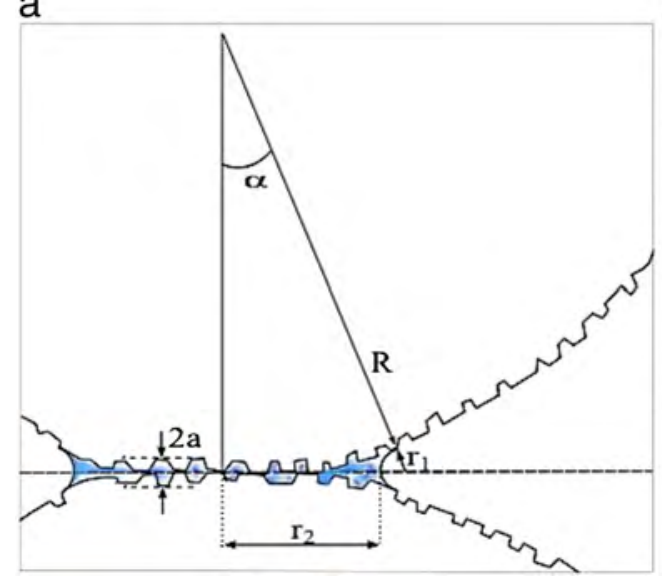

b

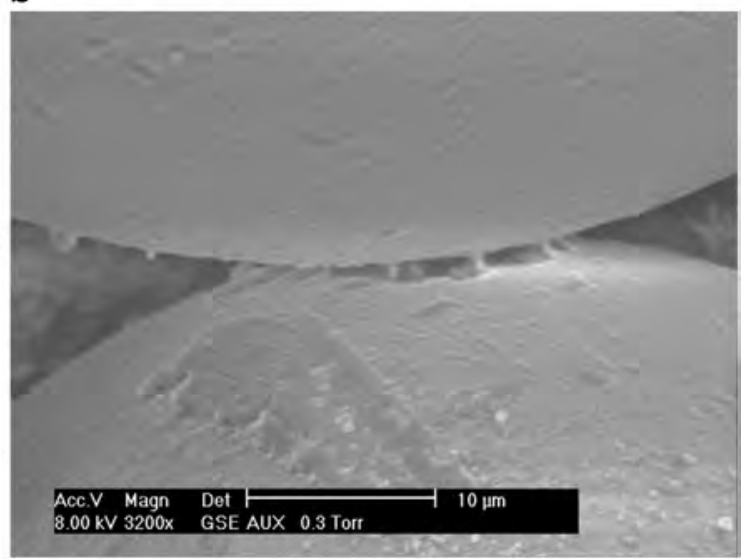

Fig. 8. a. (Left) sketch of a liquid meniscus. b. (Right) electron microscope scan image of wet glass beads ( $V_{R}=0.11 \%$ ) showing the asperities on the particle surface and at the contact point. 
for a wetting contact angle $\theta$ equals to zero, which is the case here since the glass beads are fully wetted by the $\mathrm{PEG}_{400}$. The capillary depression $\Delta P$ is given by the Laplace equation as follows

$$
\Delta P \quad \gamma\left[\frac{1}{r_{1}}-\frac{1}{r_{2}}\right]
$$

where $r_{1}$ and $r_{2}$ are the main radii of curvature (see Fig. 8a) and are given by

$r_{1} \quad R\left((1+\delta) \frac{1}{\cos \alpha}-1\right)$

$r_{2} \quad R\left(1+(1+\delta) \tan \alpha-(1+\delta) \frac{1}{\cos \alpha}\right)$

where $\alpha$ is the half filling angle (see Fig. 8a). It was estimated from the relation proposed by Rabinovich (2005) for very small magnitude of separation distance [23]:

$\alpha^{2} \quad \sqrt{2} \frac{V}{\pi R^{3}}$,

where $R$ is the harmonic mean radius used to estimate the size of the glass beads ranging from $R_{\mathrm{m}}=35 \mu \mathrm{m}$ to $R_{M}=55 \mu \mathrm{m}$ of radii $(1 / R=$ $\left.1 / 2\left(1 / R_{m}+1 / R_{M}\right)\right)$ and $V$, used to calculate the half filling angle, is the liq uid bridge volume. This volume is determined using the hypothesis of an homogeneous distribution leading to:

$$
V \quad V_{R} \frac{\frac{\pi}{6} d^{3}}{\frac{\kappa}{2}} .
$$

The capillary forces have been estimated from the above equations and introduced into Eq. (5) in order to determine the tensile strength $T_{s}$

Fig. 7 gives $T_{s}$ as a function of the volume ratio $V_{R} \%$ for various poten tial coordination numbers $\kappa$ (continuous lines), and compares these es timations with the experimental values (squares) introduced in the previous section. There is a semi quantitative agreement between the theoretical estimations and the measured values, despite some scatter ing in the experimental dataset. At high normal loading, the experimen tal observations are quantitatively close to the estimated figures when the coordination number is between 5 and 8; a slightly lower value than 9, which was the value expected for densely packed of monodis perse spheres. This is consistent with the observation of porosities higher than 0.36, indicating that the dense state is not reached, except at high normal loading and low liquid content $\left(V_{R}<0.1 \%\right)$.
This agreement suggests that the capillary contribution described by Eq. (4), has a magnitude which corresponds to the breakage of all the wetted contacts between the particles apart from the shear plane, i.e. the value that is obtained if the packed bed is sheared into two rigid blocks sliding against each other (see Fig. 9).

This phenomenon is completely different to that observed in a low applied normal stress situation, where the weak compactness of the medium prevents the simultaneous rupture of capillary bridges. The experimental tensile strength $T_{s}$ has a value whose magnitude corresponds to only one or two capillary bridges broken per particle on the shear plane. This is lower than the number of contacts predicted for loose packing. This low value suggests that in a situation of loose packing, the shear deformation is accommo dated by local rolling rearrangements which maintains some of the wet contacts between particles (see Fig. 9).

\section{Conclusion}

We have studied the shear resistance of partially wetted, monosized glass beads, in a steady state regime and for a wide range of applied normal stresses. The experimental results show that shear stress is dependent on both liquid added and applied normal stress. This de pendence appeared as a non linear shear normal stress relationship implying three different regimes as shown in this paper. In particu lar, a residual cohesion was observed at steady state for enough liquid content.

The other result of the addition of liquid is the increase in the poros ity of wet particle bed resulting from the emergence of gaps in the gran ular media due to the creation of liquid bridges. After reaching a maximum value, the porosity declines and tends to its initial value (the porosity under dry conditions).

In order to quantify the capillary forces, responsible for the shear re sistance and the variation of the porosity of the granular material, the tensile strength was evaluated using experimental data and theoretical ly estimated using the Rumpf model. The observed tensile strength is found to increase with the capillary forces as more liquid is added. How ever, the dependence of $T_{s}$ on the coordination number leads to distinc tion between two states of wet granular media. A dense configuration occurring at high applied normal stress leads to an increase in the coor dination number and then in the number of capillary bridges broken under traction. A loose configuration, at low applied normal stress, leads to a reduction in the coordination number and the preservation of some contact between particles under traction because of the rolling rearrangements. This leads to a reduction in the magnitude of the ten sile strength.

In the future, the work will be extended to other liquids, particle morphology and size distributions, which have an impact on the capil lary forces and texturization of the media.

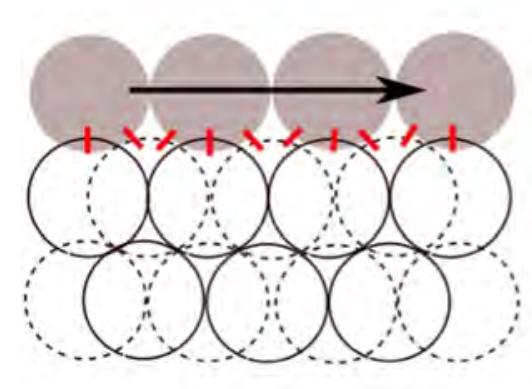

dense configuration

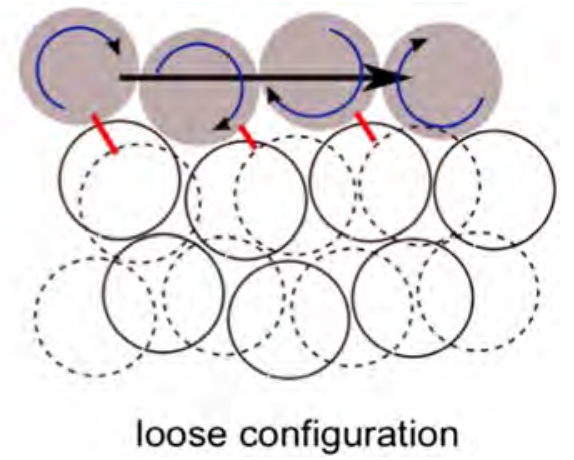

Fig. 9. Breakdown of the liquid bridges between particles apart from the shear plane and depending on the granular material configuration. 


\section{List of symbols}

A

Half separation distance $(\mu \mathrm{m})$

Area of the lid $\left(\mathrm{m}^{2}\right)$

Cohesion (Pa)

Compactness

Diameter of particles ( $\mu \mathrm{m})$

Tangential force $(\mathrm{N})$

Tangential force $(\mathrm{N})$

Against force $(\mathrm{N})$

$\mathrm{F}_{\text {cap }} \quad$ Capillary force $(\mathrm{N})$

g Graviational constant $(\mathrm{N} / \mathrm{kg})$

$\mathrm{h}_{\mathrm{t}} \quad$ Tooth height $(\mathrm{mm})$

$\mathrm{M} \quad$ Normal load $(\mathrm{kg})$

$\mathrm{N} \quad$ Normal force $(\mathrm{N})$

$\mathrm{PEG}_{400} \quad$ Polyethylene glycol 400

$\mathrm{R} \quad$ Particle radius ( $\mu \mathrm{m})$

$\mathrm{R}_{\mathrm{m}}, \mathrm{R}_{\mathrm{M}} \quad$ Minimium and maximium radius ( $\left.\mu \mathrm{m}\right)$

$r_{1}, r_{2} \quad$ Main radii of curvature ( $\left.\mu \mathrm{m}\right)$

$\mathrm{S} \quad$ Volumic liquid saturation (\%)

$\mathrm{T}_{\mathrm{s}} \quad$ Tensile strength or traction (Pa)

$\mathrm{V} \quad$ Liquid bridge volume

$\mathrm{V}_{\mathrm{R}} \quad$ Volume ratio (\%)

\section{Greek symbols}

$\alpha \quad$ Half filling angle

$\gamma \quad$ Surface tension $(\mathrm{mN} / \mathrm{m})$

$\delta \quad$ Dimensionless half separation distance

$\kappa \quad$ Coordination number

$\Delta \mathrm{P} \quad$ Capillary depression

$\Delta \tau \quad$ Standard deviation of the steady state shear stress ( $\mathrm{Pa}$ )

$\eta \quad$ Dynamic viscosity (mPa s)

$\varepsilon \quad$ Dry porosity

$\varepsilon_{\text {wet }} \quad$ Wet porosity

$\theta \quad$ Contact angle

$\mu \quad$ Friction coefficient

$\mu_{\text {dry }} \quad$ Friction coefficient of dry granular material

$\mu_{\text {wet }} \quad$ Friction coefficient of wet granular material

$\rho \quad$ Bulk density $\left(\mathrm{g} / \mathrm{cm}^{3}\right)$

$\rho_{\mathrm{L}} \quad$ Liquid density $\left(\mathrm{g} / \mathrm{cm}^{3}\right)$

$\rho_{\mathrm{s}} \quad$ True density $\left(\mathrm{g} / \mathrm{cm}^{3}\right)$

$\sigma \quad$ Normal stress $(\mathrm{Pa})$

$\tau \quad$ Shear stress (Pa)

$\tau_{\mathrm{r}} \quad$ Relative steady state shear stress (Pa)

$\tau_{\mathrm{s}} \quad$ Steady state shear stress (Pa)

$\psi \quad$ Sphericity index

$\omega \quad$ Shear cell velocity $(\mathrm{mrad} / \mathrm{s})$

\section{Acknowledgements}

The authors would like to thank M. Laurent Devriendt for his techni cal support.

\section{References}

[1] D. Hornbaker, R. Albert, I. Albert, A. Barabasi, P. Schiffer, What keeps sandcastles standing? Nature 387 (1997) 765.

[2] M. Rhodes, Introduction to particle technology, John Wiley \& Sons, Chichester, 1998.

[3] D. Newitt, J. Conway-Jones, A contribution to the theory and practice of granulation, Trans. Inst. Chem. Eng. 36 (1958) 422-442.

[4] W. Pietsch, Agglomeration Process, Wiley-VCH, Weinheim, 2002.

[5] W. Pietsch, Size Enlargement by Agglomeration, John Wiley \& Sons, Chichester, 1991.
[6] H. Rumpf, The strength of granules and agglomerates, Intersci. Publ. (1962) 379-418.

[7] N. Fraysse, H. Thomé, L. Petit, Humidity effects on the stability of a sandpile, Eur Phys. J. B 11 (1999) 615-619.

[8] L. Bocquet, É. Charlaix, F. Restagno, Physics of humid granular media, C. R. Phys. 3 (2) (2002) 207-215.

[9] D. Oulahna, R. Collet, A. de Ryck, Mechanical resistance due to shearing of partially wet granular media, Kona 30 (2012).

[10] E. Rondet, T. Ruiz, B. Cuq, Rheological and mechanical characterization of wet agglomerates processed in low shear mixer, J. Food Eng. 117 (1) (2013) 67-73.

[11] C.L. Feng, A.B. Yu, Effect of liquid addition on the packing of mono-sized coarse spheres, Powder Technol. 99 (1) (1998) 22-28.

[12] A.B. Yu, C.L. Feng, R.P. Zou, R.Y. Yang, On the relationship between porosity and interparticle forces, Powder Technol. 130 (2003) 70-76.

[13] D. Schulze, Powders and Bulk Solids, Springer-Verlag, Berlin Heidelberg, 2008

[14] D. Schulze, Time- and velocity-dependent properties of powders effecting slip-stick oscillations, Chem. Eng. Technol. 26 (10) (2003) 1047-1051.

[15] M. Lubert, A. de Ryck, Slip events and dilatancy in a sheared fine noncohesive powder, Phys. Rev. E 63 (2) (2001) 021502.

[16] R. Fisher, On the capillary forces in an ideal soil; correction of formulae given by W. B. Haines, J. Agric. Sci. 16 (1926) 492-505.

[17] J. Seville, U. Tüzün, R. Clift, Processing of Particulate Solids, Blackie, London, 1997.

[18] S. Simons, J. Seville, M. Adams, An analysis of the rupture energy of pendular liquid bridges, Chem. Eng. Sci. 49 (14) (1994) 2331-2339.

[19] M. Adams, V. Perchard, The cohesive forces between particles with interstitial liquid, Inst. Chem. Eng. Symp. 91 (1985) 147-160.

[20] K. Hotta, K. Takeda, K. linoya, The capillary binding force of a liquid bridge,, Powder Technol. 10 (1974) 231-242.

[21] W. Pietsch, Tensile strength of granular materials, Nature 217 (1968) 736.

[22] P.C.F. Moller, D. Bonn, The shear modulus of wet granular matter. EPL J. 80 (38002) (2007) 1-5.

[23] Y.I. Rabinovich, M.S. Esayanur, B.M. Moudgil, Capillary forces between two spheres with a fixed volume liquid bridge: theory and experiment. Langmuir 21 (24) (2005) 10992-10997. 